\title{
Frequency Assignment for IEEE 802.11 Wireless Networks
}

\author{
Kin K. Leung \\ Bell Labs, Lucent Technologies \\ Murray Hill, NJ 07974 \\ kin@bell-labs.com
}

\author{
Byoung-Jo "J" Kim \\ AT\&T Labs - Research \\ Middletown, NJ 07748 \\ macsbug@research.att.com
}

\begin{abstract}
The IEEE 802.11 standard specifies both radio and MAC protocol design. We observe that its CSMA protocol helps avoid much of co-channel interference by sharing radio resources in time at the potential expense of degraded network performance. Due to the coupling between the physical and MAC layers, conventional frequency allocation methods for typical cellular networks cannot be applied directly to the 802.11 networks.

In this paper, by focusing on interactions among access points, we formulate the channel assignment problem for the 802.11 network, considering the traffic load at the MAC layer, and prove that the problem is NP-complete. In light of computational complexity, a heuristic algorithm is proposed and analyzed. The algorithm is then applied to two cellular settings with known optimal assignments for verification. For one of the settings, the proposed technique generates the optimal channel assignment. As for the second case of a large network, although only a suboptimal solution is obtained by the algorithm, it is shown to be excellent. Thus, as the 802.11 networks are widely deployed, the proposed method can serve as a valuable tool for frequency planning of networks with non-uniform coverage and load.
\end{abstract}

\section{INTRODUCTION}

To meet the growing demand for wireless data services, many companies have started deploying the IEEE 802.11b wireless local-area-networks (WLAN) [199, VAM99] in places such as airports, hotels, coffee shops, etc. The 802.11 technology is particularly attractive due to its maturity and low cost. The 802.11 capability has been included as standard equipment in many laptop computers and hand-held devices. The $11 \mathrm{~b}$ WLAN supports data rates up to $11 \mathrm{Mbps}$, far exceeding that of the third generation (3G) wireless networks such as EDGE [SAE98] and W-CDMA networks [HT00].

The $802.11 \mathrm{~b}$ (and future $802.11 \mathrm{~g}$ [I03]) networks operate in the unlicensed ISM band at $2.4 \mathrm{GHz}$. Despite the relatively abundant spectrum (i.e., $75 \mathrm{MHz}$ ) at the ISM band, as the networks are deployed widely, they start to interact with each others, thus causing network performance to degrade. One way to avoid such degradation is to efficiently assign the available frequencies in the networks. This is the subject of this paper.

Let us first explain why the frequency assignment for the 802.11 network is different from that for the traditional wireless networks. In typical cellular networks including the GSM [R96] and EDGE network [SAE98], two separate radio channels, namely the traffic and control channels, are used to carry user data and control traffic, respectively. Typically, terminals access the control channels to send control information via some contention mechanism. After a base station (BS) successfully receives the information, the terminal is assigned with a specific traffic channel for transmitting its data. Existing frequency assignment methods [KN96] were devised mainly for such traffic channels. The key idea there is to keep interference to an acceptable level. In practical networks, there is no coordination among BSs in the assignment of traffic channels to terminals in different cells.

On the other hand, all user data and control information (in both directions between terminals and APs) are carried on the same physical channel in the 802.11 network. Rather, the access to the channel by multiple transmitters is coordinated by the MAC protocol - Carrier Sensing Multiple Access (CSMA) with collision avoidance. That is, a transmitter may start its transmission only if the channel is currently sensed idle. Thus, even if two closely located APs are allocated with the same channel or overlapping channels, much interference can be avoided by the CSMA protocol. In a sense, the MAC protocol provides a distributed mechanism to "coordinate" the channel access. For this reason, the 802.11 APs still operate properly, which show the robustness of the design, at the expense of increased delay (due to backoff when channel busy) and degraded network throughput. Consequently, existing frequency allocation methods that do not consider the combined effect of physical channel and MAC protocol are not applicable to the 802.11 networks. So, we propose and analyze a new frequency allocation technique in this paper.

The organization of the rest of this paper is as follows. In Section II, we formulate the frequency allocation problem, and prove it to be NP-complete. Thus, we propose and analyze a heuristic algorithm in Section III, and validate its performance in Section IV. Finally, our conclusion is in Section V.

\section{FORMULATION OF CHANNEL ASSIGNMENT}

The IEEE 802.11 [197, I99, I03] MAC protocol supports the independent basic service set (BSS), which has no connection to wired networks (i.e., ad-hoc network), as well as an infrastructure BSS, which includes an access point (AP) connecting to a wired network. The latter is similar to cellular networks with base stations replaced by AP's. We consider only the infrastructure BSS in this paper. The 802.11 MAC supports the Point Coordination Function (PCF) and the Distributed Coordination Function (DCF) [I97, OP99]. The PCF provides contention-free access, while the DCF uses the 
CSMA/CA for contention based access. The two modes can be used alternately in time. The DCF is most common in commercial 802.11 products. So, we focus on the channel assignment problem for the DCF mode here.

In North America, the ISM band at $2.4 \mathrm{GHz}$ is divided into 11 channels for the 802.11 network [OP99] where adjacent channels may partially overlap with each other. Among these 11 channels, there are 3 completely, non-overlapping ones, separated by $25 \mathrm{MHz}$ at their center frequencies. Although all 11 channels are available for allocation in 802.11 networks, experimental results reveal that partial overlapping channels can cause enough interference [M01]. Thus, we consider the assignment of non-overlapping channels here, although our approach can be extended to consider allocation of overlapping channels with appropriate weighting factors.

As a first approach, let us focus on the transmission by the APs in the $802.1 \mathrm{~b}$ network. Such a focus is appropriate for typical office and Internet applications, because the bandwidth consumption for downlink (i.e., from AP to terminal) influences application performance than that for uplink (i.e., from terminal to AP). Let the network have $M$ APs, indexed from 1 to $M$. By the CSMA protocol, an AP with traffic ready to transmit, first determines if the assigned channel is busy or idle. That is, if the AP detects that the received power within the channel is greater than $\alpha \mathrm{mW}$ (typically about $-76 \mathrm{dBm}$ or lower [197]), the channel is declared to be busy. ${ }^{*}$ Otherwise, it is idle.

Clearly, the channel busy status can be due to a single transmitting AP or a group of multiple APs transmitting simultaneously. For efficient frequency assignment, let us classify the interferers for each AP. Specifically, for each AP $i$, let $\mathrm{C}_{\mathrm{i}}(1)$ denote a set of interfering APs where transmission by any one AP in the set can cause enough interference for AP $i$ to detect channel busy. The APs in the set $\mathrm{C}_{\mathrm{i}}(1)$ are called class-1 interferers for AP $i$. Likewise, let $\mathrm{C}_{\mathrm{i}}(2)$ be a set of pairs of two interfering APs where transmission by any pair of APs in the set can cause AP $i$ to sense channel busy. APs in $\mathrm{C}_{\mathrm{i}}(2)$ are called class-2 interferers. In general, to determine the interferer sets $\mathrm{C}_{\mathrm{i}}(1)$ and $\mathrm{C}_{\mathrm{i}}(2)$ for each $\mathrm{AP} i$ requires measurements or estimates of signal path loss between each pair of APs in the network. Let $p_{j}$ and $h_{i j}$ denote the transmission power at $\mathrm{AP} j$ and the signal path loss from $\mathrm{AP} j$ to AP $i$. If $\mathrm{AP} j$ belongs to $\mathrm{C}_{\mathrm{i}}(1)$, it requires

$$
h_{i j} p_{j} \geq \alpha \text {. }
$$

Similarly, if AP pair $m$ and $n$ belong to $\mathrm{C}_{\mathrm{i}}(2)$, we have

\footnotetext{
* Besides a received power threshold, the 802.11 standard also allows a commonly implemented option of determining channel busy by detecting a valid 802.11 signal (carriersense), which is often possible in a same channel around -95 $\mathrm{dBm}$ with little other channel impairments. Without loss of generality, we consider the method by a received power threshold in this paper, since the carrier-sense can be translated into an equivalent power level with minor simplifying assumptions.
}

$$
h_{i m} p_{m}+h_{i n} p_{n} \geq \alpha .
$$

Note that the transmission power in (1) and (2) are assumed to be fixed in this paper and channel assignment with dynamic power control is a topic for future study.

Similarly, we can define class-3 or even higher classes of interferers. However, due to the contention nature of the CSMA protocol, the traffic load on each channel (i.e., the probability of transmission at a given AP) cannot be too high. Thus, the probability of having class- 3 interferers, which require simultaneous transmission at all 3 interfering APs that could not hear each other, is much smaller relative to that of the class- 1 and 2 interferers. For simplicity, we only consider class- 1 and 2 interferers here.

Let us define additional notation. Let $\rho_{\mathrm{i}}$ be the offered traffic load for AP $i$ in terms of channel utilization without interference from any source. There are totally $N$ (nonoverlapping) channels, indexed by 1 to $N$, available for allocation. As pointed out above, $N=3$ for the $802.11 \mathrm{~b}$ network. Without loss of generality, we assume that each AP is assigned with one and only one channel. Further, we denote $X_{i j}=1$ if AP $i$ is assigned with channel $j$ and 0 otherwise. Since the CSMA protocol prohibits APs from transmitting when the channel is sensed busy, we define the effective channel utilization $U_{i}$ as the fraction of time at which the channel can be sensed busy or is used for transmission by AP $i$. That is,

$$
\begin{aligned}
U_{i} \equiv & \rho_{i}+\sum_{k=1}^{N} X_{i k}\left[\sum_{j \in C_{i}(1)} \rho_{j} X_{j k}\right. \\
& \left.+\sum_{(m, n) \in C_{i}(2)} \rho_{m} \rho_{n} X_{m k} X_{n k}\right] \cdot
\end{aligned}
$$

This definition can be interpreted as follows. The first term on the right hand side is the offered load associated with AP $i$. The first summation term inside the brackets represents the total traffic load of all class-1 interfering APs that are assigned with the same channel as AP $i$. This is so because according to the CSMA protocol and the detection threshold $\alpha$ in use, AP $i$ senses channel busy when any one of its class- 1 interferers transmits on the same channel. Similarly, the last summation term represents the effective channel utilization due to class- 2 interferers if they use the same channel.

Our objective function for the channel assignment is to minimize the utilization at the most stressed bottleneck AP:

$$
\text { Minimize } \operatorname{Max}\left\{U_{1}, U_{2}, \ldots, U_{M}\right\}
$$

over the assignment indicator $\left\{X_{i j}\right\}$. The optimal assignment is feasible if its objective function value is less than 1 . That is so because to maintain channel stability, we require

$$
U_{i}<1
$$

for all AP $i=1$ to $M$. In fact, the RHS of (5) can be a value less than 1 to account for CSMA overhead and stability margin.

Theorem 1. The optimization problem with the objective function in (4) is NP-complete. 
Proof.. Consider the multiprocessor scheduling problem A5.2 [GJ79, p.238] with $m$ processors and $T$ tasks. Each task $i$ requires $l(i)$ time units to process. The optimal scheduling is to assign tasks to processors such that the maximum total processing time on all processors is minimized. Our proof is to reduce the scheduling problem, which is NP-complete, to the frequency assignment problem as follows. Each processor in the scheduling problem is treated as a radio frequency in the assignment problem. So, $N=m$. Each task is represented by an AP (thus $M=T$ ) and each processing time $l(i)$ becomes the traffic load $\rho_{\mathrm{i}}$ for the corresponding AP $i$. To complete the reduction, all APs are treated as class-1 interferers to each other. Define that $V_{k}$ is the total traffic load associated with all APs assigned with frequency $k$ for each $k=1$ to $N$. Clearly, minimizing the maximum total processing time on all processors is identical to minimizing $\left\{V_{k}\right\}$ for all $k=1$ to $N$. Note that $V_{k}=U_{i}$ if $X_{i k}=1$ for each $k$ and $i$. Further, $U_{i}=U_{j}$ if $X_{i k}=X_{i k}$ for some frequency $k=1$ to $N$ because all APs $i$ and $j$ are class- 1 interferers to each other. Thus, the proof is completed by observing that maximizing $\left\{V_{k}, k=1\right.$ to $\left.N\right\}$ is identical to maximizing $\left\{\mathrm{U}_{k}, k=1\right.$ to $\left.M\right\}$ in (4).

In light of NP-completeness, it is unlikely that an efficient method exists for the optimal channel assignment. Thus, we propose a heuristic method and study its effectiveness below.

\section{A Heuristic METHOD FOR ChANNEL ASSIGNMENT}

We propose a heuristic algorithm that attempts to minimize the effective channel utilization for the bottleneck AP in (4). The new algorithm makes use of the following known parameters: offered traffic load $\rho_{\mathrm{i}}$ and its interferer sets $\mathrm{C}_{\mathrm{i}}(1)$ and $\mathrm{C}_{\mathrm{i}}(2)$ for each $\mathrm{AP} i$. We outline the algorithm as follows:

1. Generate a random, initial channel assignment for the network, which is treated as the best assignment obtained so far. Let the maximum effective channel utilization for the assignment be denoted by $V$ (i.e., $V=\max \left\{U_{i}\right\}$ ).

2. Based on the best assignment, identify the AP (say $i$ ) with the highest effective channel utilization. In case of tie, one such AP $i$ is chosen randomly as the "bottleneck."

3. For the bottleneck AP $i$, identify its current assigned channel, say $k$. For each available channel $n$ from 1 to $N$ with $n \neq k$ and each co-channel AP (say $j$ ) in $\mathrm{C}_{\mathrm{i}}(1)$ (i.e., those APs in the set that have been assigned with channel $m$ ), temporarily modify the channel assignment by reassigning only AP $j$ with channel $n$. Based on (3), recompute the maximum effective channel utilization, denoted by $W_{j n}$, for the new assignment. After completing such testing for all such $n$ and $j$, let $W$ be the minimum among all the $W_{j n}$ 's.

4. Compare $W$ with $V$ and perform the following:

a. If $W<V$, then replace $V$ by $W$ and record the associated new assignment as the new best solution (i.e., to finalize the channel change for one AP that minimizes the objective function the most - a greedy step). Continue with Step 2.

b. If $W=V$, then with a pre-specified probability $\delta$, replace $V$ by $W$ and record the new assignment as the best solution. Continue with Step 2. c. If $W>V$, a local optimum has been reached (i.e., the best assignment obtained so far is the local suboptimal solution). Continue with Step 5 .

5. Repeat Steps 1 to 4 with a number of random, initial assignments. The final solution is chosen to be the best, according to (4), among the local suboptimal assignments.

6. Test if constraints (5) for all APs are satisfied for the final assignment. If so, the final assignment is feasible. Otherwise, it is considered that no feasible solution exists for the network under consideration.

Clearly, this algorithm does not explicitly consider the constraints (5). However, focusing on (4) by the algorithm is appropriate since minimizing the maximum $U_{i}$ automatically enhances the chance of satisfying constraints (5) for all APs. Now, let us consider a property of the proposed algorithm.

\section{Theorem 2. With $1>\delta>0$ in Step 4, the heuristic algorithm} does not have infinite looping.

Proof. Since the number of APs $M$ and available channels $N$ are finite, Steps 2 and 3 can be completed in a finite amount of time. The only possibility that the algorithm has an infinite loop is that Steps 2 to 4 are executed repeatedly without stop. Assume that such looping can happen and the $V$ value after the $m$-th execution (iteration) of Step 4 be denoted by $V_{m}$. To proceed, let $\delta=0$ in Step $4 \mathrm{~b}$ for a moment. To form the infinite looping, we must have $V_{1}>V_{2}>\ldots>V_{m}$ with $m \rightarrow \infty$. With both $M$ and $N$ being finite, there are only a finite number of all possible channel assignments. Since each new assignment finalized by Step 4a has a unique maximum effective channel utilization, it is thus impossible that $m$ goes to infinity. That is, Step $4 \mathrm{c}$ must be reached after a finite amount of processing.

Let us assume that infinite looping is possible with $1>\delta>0$. Based on the above argument, we now must have $V_{l}>\ldots>V_{i}=V_{i+1}>\ldots>V_{j}=V_{j+1}>\ldots V_{m}$ with $m \rightarrow \infty$ for some $i$ and $j$. Since the argument above has already ruled out the possibility of having subsequences of $V_{i}$ 's of infinite length between two $=$ sign on this list, it must contain an infinite number of $=$ sign. Since each $=$ sign corresponds to an execution of Step $4 \mathrm{~b}$ with probability $\delta$, the probability of executing this step for an infinite number of time is thus zero. Hence, the infinite looping cannot exist.

We remark that based on the proof, the algorithm can exclude Step $4 \mathrm{~b}$ and treat the case of $W=V$ as reaching a local optimum as part of Step 4c, without causing any infinite looping. However, our numerical experience reveals that Step $4 \mathrm{~b}$ helps explore various assignments for enhanced results, especially when there are multiple bottleneck APs for the channel assignment under consideration.

Since heuristics is involved in the proposed algorithm, achieving the optimal solution is not guaranteed. Now, let us quantify the quality of the suboptimal solution generated by the algorithm. Toward this goal, we observe that Step 3 basically tests out various channel assignments to identify a better solution. As the algorithm is executed for a given initial, random assignment, let $Y_{0}, Y_{l}, Y_{2}, \ldots, Y_{m}$ denote the (random) sequence of the maximum effective channel utilization 
associated with the channel assignments under testing. We denote that $Y_{0}$ is the quantity for the initial, random assignment. Based on the $Y_{i}$ sequence, we construct another sequence $Z_{0}, Z_{1}, Z_{2}, \ldots, Z_{n}$ as follows.

1. We start with $Z_{0}=Y_{0}$ and set $i=0$.

2. For each $j=1,2, \ldots, m$, compare $Y_{j}$ with $Z_{i}$. If $Z_{i}>Y_{j}$, then we set $i=i+1$ and $Z_{i}=Y_{j}$. Otherwise, repeat Step 2 for the next $j$ value.

In essence, the sequence $\mathrm{Z}_{\mathrm{i}}$ 's is constructed by examining $Y_{j}$ one by one. We start with $Z_{0}=Y_{0}$ and $Y_{j}$ is added as the last element in the $Z_{i}$ sequence only if $Y_{j}$ is less than $Y_{i}$ for all $i<j$ (or equivalently, $Y_{j}$ is less than $Z_{i}$, the last element in the current sequence). Clearly, the sequence $Z_{i}$ is monotonic strictly decreasing. Physically, $Z_{i}$ 's represent the sequence of the maximum effective channel utilization for an improved assignment finalized by Step $4 \mathrm{a}$ or $4 \mathrm{~b}$ that yields a maximum utilization lower than any assignments examined by the algorithm so far in the search process.

Recall that the algorithm is repeated for a given number (say $K$ ) of initial random assignments. For each initial assignment, we can obtain one such sequence $Z_{i}$ 's as discussed above. Note that the sequences associated with different initial assignments have different lengths and are mutually independent of each other (although elements in the same sequence are dependent). Furthermore, when the algorithm eventually stops, assume that it has encountered a total of $n$ improved assignments (i.e., improved over those examined earlier and derived from the same initial assignment), which is the sum of lengths of the sequences $Z_{i}$ 's minus $K$.

One can view that the maximum effective channel utilization for all possible assignments for the given network has a probability distribution. Let $T_{\pi}$ be the maximum utilization for the top- $\pi$-fraction of assignments (e.g., the top 0.001 percentile assignments). Hence, for a random assignment with its maximum utilization $Z_{0}$, we have

$$
P\left[Z_{0} \leq T_{\pi}\right]=\pi \text {. }
$$

Let $Q_{\pi}$ be the probability that the final suboptimal solution by the algorithm falls within the top- $\pi$-fraction of assignments.

Theorem 3. If the algorithm have encountered a total of $n$ improved assignments at the completion of its execution, then

$$
Q_{\pi}>1-(1-\pi)^{n+1}
$$

Proof. First consider the case of encountering $n$ improved assignments for one initial, random assignment. By definition,

$$
Q_{\pi}=P\left[\min _{i} Z_{i} \leq T_{\pi}\right]=1-P\left[\min _{i} Z_{i}>T_{\pi}\right] .
$$

The event of $\left(\min Z_{\mathrm{i}}>\mathrm{T}_{\pi}\right)$ in the above is identical to having $\mathrm{Z}_{\mathrm{o}}>\mathrm{T}_{\pi}, \mathrm{Z}_{1}>\mathrm{T}_{\pi}, \ldots$, and $\mathrm{Z}_{\mathrm{n}}>\mathrm{T}_{\pi}$. Given that $Z_{i}$ 's are a strictly decreasing (random) sequence, we have

$$
\begin{aligned}
& P\left[Z_{0}>T_{\pi} \wedge Z_{1}>T_{\pi} \wedge \ldots \wedge Z_{n}>T_{\pi}\right] \\
& \quad<P\left[Z_{0}>T_{\pi} \wedge Z_{0}^{1}>T_{\pi} \wedge \ldots \wedge Z_{0}^{n}>T_{\pi}\right]
\end{aligned}
$$

Where $Z_{0}{ }^{i}$ is a random variable independently drawn from the same distribution for $Z_{0}$ for $i=1$ to $n$. One can obtain (10) by replacing $Z_{i}$ on the left hand side by $Z_{0}{ }^{i}$ on the right side for one $i$ at a time. Since the $Z_{0}^{i}$ variables are independent,

$$
P\left[Z_{0}>T_{\pi} \wedge Z_{0}^{1}>T_{\pi} \ldots Z_{0}^{n}>T_{\pi}\right]=\left\{P\left[Z_{0}>T_{\pi}\right]\right\}^{n+1}
$$

Using the definition in (7), substituting (11) into (10) and then inputting into (9) yields (8). The case with multiple initial random assignments is proven by exploiting the property that the sequences $Z_{i}$ 's associated with different initial assignments are mutually independent.

\section{METHOD VALIDATION}

For validation, we apply the proposed algorithm to two cellular layouts for which the optimal assignment is known. Specifically, the settings correspond to a network with 7 and 37 cells in Fig. 1 and 2, respectively. A cell is divided into 3 sectors, each of which is represented by a clover-leaf hexagon and served by an access point (AP) at the center of the cell (a dot in the figures). Each AP antenna has a beamwidth of $60^{\circ}$ and points toward an appropriate direction to serve the associated sector. Thus, there are 21 and 111 APs in Fig. 1 and 2 , with 3 APs in each cell co-located at the cell center. The antenna gain has a parabolic shape; that is, a $3 \mathrm{~dB}$ drop relative to the front direction occurs at the half bandwidth angle. Antenna gain in the front direction is about $15 \mathrm{dBi}$. Any

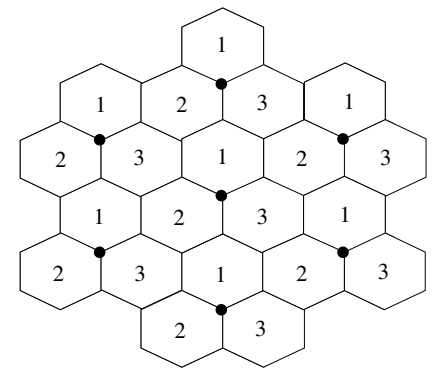

Fig. 1. Assignment for Network with 7 Cells and 21 APs

direction beyond a threshold angle in clockwise or anticlockwise direction suffers a given, fixed attenuation relative to the gain at the front direction, which is called the front-toback (FTB) ratio. The FTB is set to be $25 \mathrm{~dB}$.

Recall that only the AP-to-AP interaction is considered in our current formulation. The radio link between any pair of APs is characterized by a path-loss model with an exponential of 3.5. Cell radius is $1 \mathrm{Km}$ and the path loss at $100 \mathrm{~m}$ from the cell center is $-73 \mathrm{~dB}$. Transmit power for each AP antenna is 30 $\mathrm{dBm}$ (or $1 \mathrm{~W}$ ). To ensure that the optimal assignment is known, shadowing and fast fading are not considered and all APs have identical amount of offered traffic. In addition, the channel-busy detection threshold $\alpha$ is set to be $2.5 \mathrm{e}-3 \mu \mathrm{W}$ (or $86 \mathrm{dBm})$. As pointed out earlier, there are 3 non-overlapping channels available for assignment in the $2.4 \mathrm{GHz}$ ISM band. Based on the parameter settings for both 7 and 37-cell networks, the optimal assignment is the traditional frequency 
reuse of 3 [L89]. That is, no adjacent sectors use the same channel.

When the proposed algorithm was applied to the network with 7 cells and 21 APs, the algorithm was able to generate the optimal channel assignment, based on 50 initial random assignments. The optimal assignment with channels 1 to 3 assigned to various sectors (APs) is shown in Figure 1.

As for the network with 37 cells and 111 APs, the proposed algorithm was not able to yield the optimal assignment. The suboptimal solution obtained from the algorithm using 1,000 initial random assignments is presented in Figure 2. The 3 channels are represented in red, blue and green color. As shown in the figure, most of the sectors (APs) use a channel different from those in adjacent sectors. In the worst case, at most two adjacent sectors share the same channel. In the search process, the algorithm encountered and finalized a total of 505,363 improved assignments. Based on the analysis in Section III, with a probability higher than $99.4 \%$, the suboptimal solution in Figure 2 falls within the top 0.001th percentile, which is quite acceptable. In this case, the algorithm took 9 minutes to run on a SUN Sparc workstation. An exhaustive search must examine in the order of $10^{52}$ assignments.

Clearly, the advantage of this algorithm is evident when applied to a large WLAN layout with non-uniform traffic loads over the network and other constraints where a good frequency assignment, let alone an optimal one, is not as obvious as the examples used here for verification purposes.

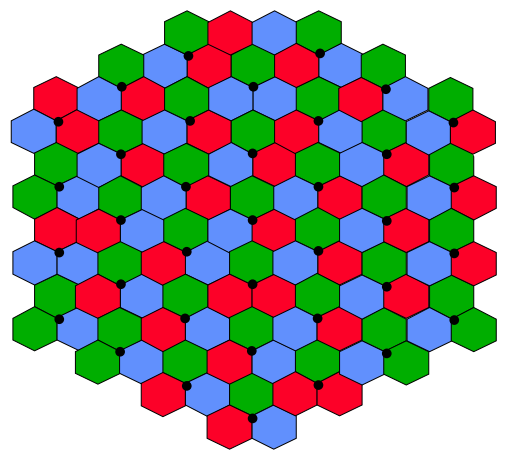

Fig. 2. Assignment for Network with 37 Cells and 111 APs

\section{CONCLUSION}

We observe that the CSMA protocol in use in 802.11 helps avoid much of co-channel interference at the possible expense of degraded network throughput. Due to the coupling between the physical and MAC layers, conventional frequency allocation methods, typically for traffic channels on cellular networks, cannot be applied directly to the 802.11 networks.

In this paper, by focusing on interactions among access points (APs), we formulate the channel assignment problem for the 802.11 network, which is then proven to be NPcomplete. In light of computational complexity, a heuristic algorithm is proposed and analyzed. Specifically, the algorithm was shown to be loop-free. A metric to assess the quality of the solution generated by the algorithm is proposed and proven. For validation, the algorithm was applied to two cellular settings with known optimal assignments. For one of the settings, the proposed algorithm was able to generate the optimal channel assignment. As for the second case of a large network, although only a suboptimal solution was obtained by the algorithm, its quality has been shown to be excellent. Therefore, as the 802.11 networks are widely deployed, the proposed algorithm can serve as a valuable tool for frequency planning of WLAN networks.

In terms of future work, we are extending the proposed approach to consider non-uniform transmission power by the APs, uplink traffic, and adaptive channel assignment to meet the time fluctuation of traffic load at various APs.

\section{ACKNOWLEDGMENTS}

We thank Bruce McNair for sharing his results with us and for his discussion. Thanks are also due to Leonard Cimini, Paul Henry and Zoran Kostic for their discussion.

\section{REFERENCES}

[GJ79] M.R. Garey and D.S. Johnson, Computer and Intractability: A guide to the Theory of NPCompleteness, W.H. Freeman and Company, San Francisco, 1979.

[HT00] H. Holma and A. Toskala (Ed.), WCDMA for UMTS, John Wiley \& Sons, New York, 2000.

[I97] IEEE 802.11, "Wireless LAN Medium Access Control (MAC) and Physical Layer (PHY) Specification," 1997.

[199] IEEE 802.11b, "Part 11: Wireless LAN Medium Access Control (MAC) and Physical Layer (PHY) Specification: High-Speed Physical Layer Extension in the $2.4 \mathrm{GHz}$ Band," 1999.

[I03] IEEE 802.11g, "Wireless LAN Medium Access Control (MAC) and Physical Layer (PHY) Specifications: Higher Speed Physical Layer (PHY) Extension to IEEE 802.11b," 2003.

[KN94] I. Katzela and M. Naghsineh, "Channel assignment schemes for cellular mobile telecommunication systems: a comprehensive survey," IEEE Personal Commun., Vol: 3, June 1996, pp. 10-31.

[L89] W.C.Y. Lee, Mobile Cellular Telecommunications Systems, McGraw-Hill, New York, 1989.

[M01] B. McNair, private communications, 2001.

[OP99] B. O'Hara and A. Petrick, IEEE 802.11 Handbook, IEEE Press, New York, 1999.

[R96] T. S. Rappaport, Wireless Communications: Principles and Practice, New York: IEEE Press and Prentice Hall, 1996.

[SAE98] P. Schramm, et. al., "Radio Interference Performance of EDGE, a Proposal for Enhanced Data Rates in Existing Digital Cellular Systems," Proc. of IEEE VTC, Ottawa, Canada, May 1998, pp. 1064-1068.

[VAM99] R. van Nee, et. al., "New High-Rate Wireless LAN Standard," IEEE Commun. Mag., Dec. 1999, pp. 82-88. 